\title{
DNA Damage Response is Prominent in Ovarian High-Grade Serous Carcinomas, Especially Those with Rsf-1 (HBXAP) Overexpression
}

\author{
Malti Kshirsagar, ${ }^{1}$ Wei Jiang, ${ }^{1,2}$ and Ie-Ming Shih ${ }^{1}$ \\ ${ }^{1}$ Department of Pathology, Johns Hopkins Medical Institutions, Baltimore, MD 21231, USA \\ ${ }^{2}$ Department of Pathology, Cleveland Clinics, Cleveland, OH 44195, USA
}

Correspondence should be addressed to Ie-Ming Shih, ishih@jhmi.edu

Received 14 May 2011; Revised 29 July 2011; Accepted 11 August 2011

Academic Editor: Kentaro Nakayama

Copyright ( $\odot 2012$ Malti Kshirsagar et al. This is an open access article distributed under the Creative Commons Attribution License, which permits unrestricted use, distribution, and reproduction in any medium, provided the original work is properly cited.

\begin{abstract}
DNA damage commonly occurs in cancer cells as a result of endogenous and tumor microenvironmental stress. In this study, we applied immunohistochemistry to study the expression of phosphorylated Chk2 (pChk2), a surrogate marker of the DNA damage response, in high grade and low grade of ovarian serous carcinoma. A phospho-specific antibody specific for threonine 68 of Chk2 was used for immunohistochemistry on a total of 292 ovarian carcinoma tissues including 250 high-grade and 42 low-grade serous carcinomas. Immunostaining intensity was correlated with clinicopathological features. We found that there was a significant correlation between pChk2 immunostaining intensity and percentage of pChk2 positive cells in tumors and demonstrated that high-grade serous carcinomas expressed an elevated level of pChk2 as compared to low-grade serous carcinomas. Normal ovarian, fallopian tube, ovarian cyst, and serous borderline tumors did not show detectable pChk2 immunoreactivity. There was no significant difference in pChk2 immunoreactivity between primary and recurrent high-grade serous carcinomas. In high-grade serous carcinomas, a significant correlation $(P<0.0001)$ in expression level (both in intensity and percentage) was found between pChk2 and Rsf-1 (HBXAP), a gene involved in chromatin remodeling that is amplified in high-grade serous carcinoma. Our results suggest that the DNA damage response is common in high-grade ovarian serous carcinomas, especially those with Rsf-1 overexpression, suggesting that Rsf-1 may be associated with DNA damage response in high-grade serous carcinomas.
\end{abstract}

\section{Introduction}

Ovarian carcinomas comprise a diverse group of neoplasms that demonstrate distinct clinicopathological features and unique molecular genetic aberrations with respect to different histologic subtypes $[1,2]$. Based on clinicopathological and molecular genetic features, we have previously proposed that ovarian carcinoma can be classified into two major types, type I (low-grade serous, low-grade endometrioid, clear cell, and mucinous carcinomas) and type II (mainly high-grade serous carcinomas) [3]. Given the fact that different subtypes of ovarian tumors develop along distinct molecular pathways, we asked if the DNA damage response (DDR) is different among high-grade and low-grade serous carcinomas, the prototypes of type II and type I tumor, respectively.
It has been well established that the DDR pathway is activated by endogenous and environmental cellular stress that is associated with DNA damage and has profound effects on determining cell fate. DDR signaling has been reported to be associated with several types of human cancer including colorectal, pancreatic, and oral squamous cell carcinomas [4-6]. Given its critical role in tumor development, it has been proposed that harnessing the activity of DDR pathways may improve cancer treatment outcome after cytotoxic chemotherapy and irradiation therapy [7]. Molecularly, DDR is initiated by the rapid recruitment of several nuclear proteins involved in the repair process to the site of DNA damage to form a complex, which acts to repair DNA damage and promote cell survival. DDR is mediated by a signal transduction cascade involving the ataxia telangiectasia mutated (ATM-) check point kinase 2 (Chk2)-p53 axis 


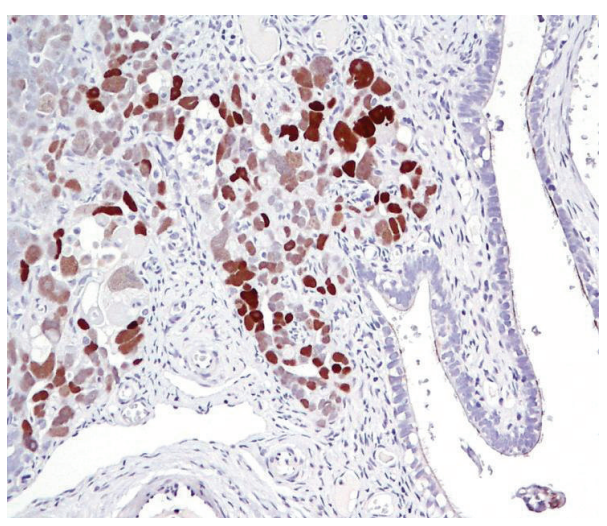

(a)

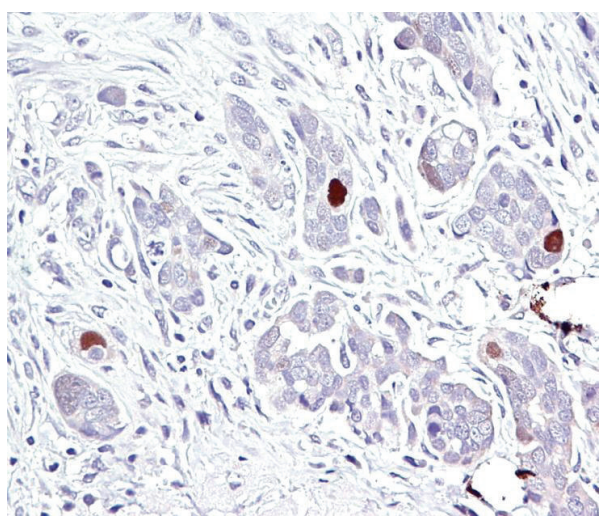

(c)

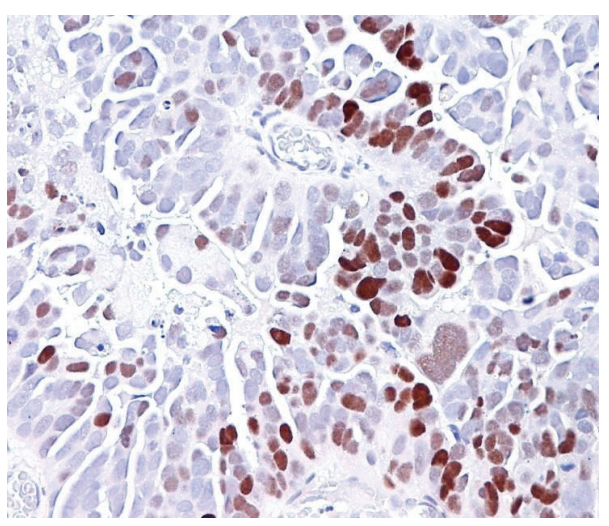

(b)

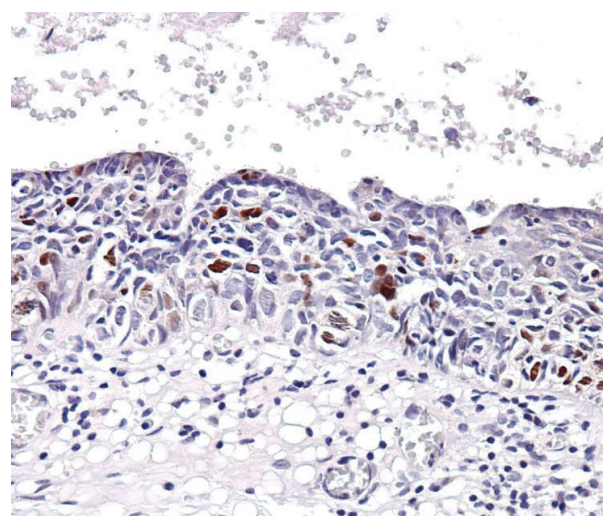

(d)

FIGURE 1: pChk2 immunoreactivity in representative high-grade serous carcinomas. (a) A high-grade serous carcinoma shows relatively diffuse positive staining for pChk2. In contrast, pChk2 immunoreactivity is undetectable in the adjacent fallopian tube epithelium. (b) At a higher magnification, pChk2 immunoreactivity is exclusively present in the nuclei of tumor cells. (c) Another high-grade serous carcinoma shows focal pChk2 staining in cancer cells. (d) A serous tubal intraepithelial carcinoma, a presumable precursor of high-grade serous carcinoma, contains pChk2 positive cells in the lesion.

TABLE 1: Immunostaining intensity of pChk2 in ovarian serous tumors, normal ovaries, and fallopian tubes.

\begin{tabular}{lcccccc}
\hline Staining intensity & $\begin{array}{c}\text { Low-grade } \\
\text { SC }\end{array}$ & $\begin{array}{c}\text { High-grade } \\
\text { SC (primary) }\end{array}$ & $\begin{array}{c}\text { High-grade } \\
\text { SC (recurrent) }\end{array}$ & SBT & Serous cystadenoma & Normal ovaries and FTE \\
\hline 0 & $31(73.8 \%)$ & $47(29.6 \%)$ & $28(30.7 \%)$ & $16(100 \%)$ & $10(100 \%)$ & $5(100 \%)$ \\
$1+$ & $5(11.9 \%)$ & $37(23.3 \%)$ & $15(16.5 \%)$ & 0 & 0 & 0 \\
$2+$ & $3(7.1 \%)$ & $39(24.5 \%)$ & $18(19.8 \%)$ & 0 & 0 \\
$3+$ & $3(7.1 \%)$ & $36(22.6 \%)$ & $30(33 \%)$ & 0 & 0 & 0 \\
\hline
\end{tabular}

SC: serous carcinoma; SBT: serous borderline tumor; FTE: fallopian tube epithelium.

$[8,9]$. In this cascade, Chk2 plays a pivotal role; ATM phosphorylates Chk2 to generate pChk2, the active form of Chk2, which then activates several downstream pathways, leading to cell cycle arrest through p53, BRCA1, Cdc25A, and Cdc25C phosphatase $[10,11]$. Because threonine 68 of Chk2 is phosphorylated at sites of DNA strand breaks and the specific antibody that binds pChk2 at threonine 68 is available [12], pChk2 immunoreactivity has been used in many studies as a surrogate tissue biomarker for DDR [4-6, 13, 14]. In fact, it has been reported that Chk2 with Thr 68 phosphorylation was detected in more than $50 \%$ of primary untreated lung and breast tumor specimens [15].
In this study, we address three main questions: whether there is a difference in the level of DDR between highgrade and low-grade serous carcinoma; whether recurrent high-grade serous carcinomas have an altered DDR as compared to their primaries; whether there is a significant correlation in the expression levels between pChk2 and Rsf-1 (HBXAP), a gene that is frequently upregulated in highgrade serous carcinoma and participates in generating DNA damage. The Rsf-1 gene, located at ch11q13.5, is frequently amplified, and its expression is upregulated in most highgrade serous carcinomas but not in type I tumors [16-18]. Here we applied pChk2 immunohistochemistry to assess 


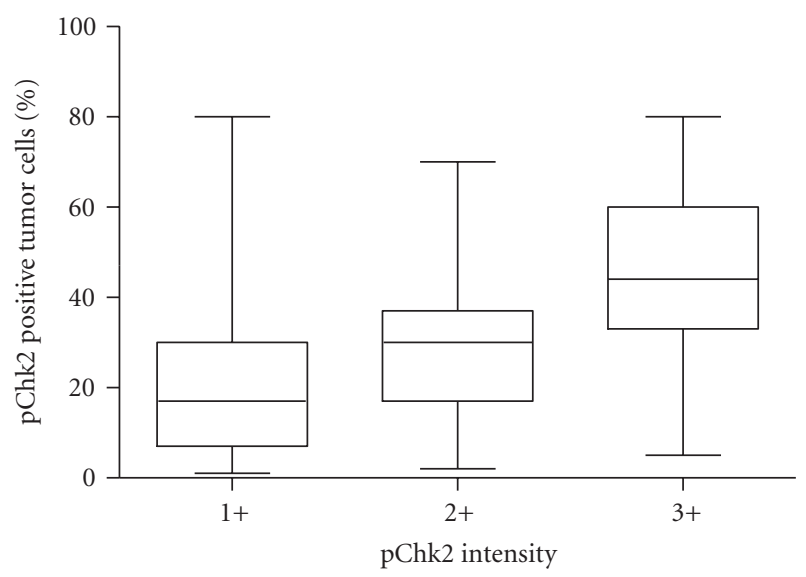

FIgURE 2: Box plot for the correlation of pChk2 immunostaining intensity and percentage of tumor cells showing pChk2 staining among 206 ovarian tumor tissues. The percentage of $\mathrm{pChk} 2$ positive cells correlates with intensity grade: percent pChk 2 positive cells in $3+$ cases $>2+$ cases $>1+$ cases $(P<0.0001$, nonparametric one-way ANOVA test).

the levels of DDR in high-grade serous carcinoma, lowgrade serous carcinoma, serous borderline tumor, and serous cystadenoma. Comparing the levels of DDR in cancer cells should help understand the pathogenesis of different subtypes of ovarian serous carcinoma.

\section{Methods}

An antibody specific for threonine 68 (Thr 68) of Chk2 (Cell Signaling, Danvers, Mass) was used for immunohistochemistry on ovarian carcinoma tissues at a dilution of $1: 200$. Phosphorylation of Chk2 at Thr 68 is a prerequisite for full activation by ATM (3). The specificity of the antibody was reported in a previous study [19]. A total of 292 ovarian carcinomas were analyzed, including 250 high-grade serous carcinomas (159 primary and 91 recurrent tumors) and 42 low-grade serous carcinomas. In addition, normal ovaries and fallopian tubes from 5 patients, 10 serous cystadenomas, and 16 serous (atypical proliferative) borderline tumors were also analyzed. All the ovarian tumors tissues except 14 lowgrade serous tumors were arranged in tissue microarrays in triplicate ( $1.5 \mathrm{~mm}$ core) to facilitate immunohistochemistry. pChk2 immunoreactivity was semiquantitatively scored by two pathologists using a four-tiered grading system (0 to $3+$ ) for intensity and percentage for prevalence of positive cells. Correlations of intensity and percentage of pChk2 with clinical data including grade (Chi square), diseasefree interval, and overall survival (Kaplan-Meier curves) were determined. A monoclonal anti-Rsf-1 antibody, clone 5H2/ E4 (Upstate, Lake Placid, NY), was used for immunostaining in 75 high-grade ovarian serous carcinomas arranged in tissue microarrays. Rsf-1 immunoreactivity was semiquantitatively scored by two pathologists independently using a five-tier grading system (0 to $4+$ ) as previously described [17]. A nonparametric Kruskal-Wallis test was used to determine the statistical significance of correlation between
pChk2 expression (both intensity and percentage) and Rsf-1 immunostaining intensity.

\section{Results}

pChk2 immunoreactivity was exclusively localized in the nuclei of tumor cells (Figure 1). In general, the intensity of pChk2 and the percentage of pChk2 positive cells varied among tumor samples (Figures $1(\mathrm{a})-1(\mathrm{c})$ ) but were highly correlated. The percentage of positive cells in $3+$ cases was greater than that in $2+$ cases which in turn was higher than that in $1+$ cases $(P<0.0001$, nonparametric one-way ANOVA test) (Figure 2). For example, among high-grade serous carcinomas, using a percentage of $30 \%$ as an arbitrary cutoff, $77 \%$ of $3+$ tumors, $33 \%$ of $2+$ tumors, and $21 \%$ of $1+$ tumors contained pChk 2 positive cells in more than $30 \%$ of cells. The pChk2 immunoreactivity in different types of ovarian serous tumors and normal tissues was summarized in Table 1. Comparing low-grade to high-grade carcinomas, we found that $66(26.4 \%)$ of 250 high-grade serous carcinomas, including primary and recurrent tumors, showed intense pChk 2 immunoreactivity (3+) while only $3(7.1 \%)$ of 42 low-grade serous carcinomas had immunostaining scores of $3+$. If the cutoff of $<2$ versus $\geq 2$ was used, 123 of 250 (49.2\%) type II carcinomas and 6 (14.2\%) of 42 low-grade serous carcinomas had scores $\geq 2$. As compared to low-grade carcinomas, high-grade serous carcinomas demonstrated a statistically higher frequency of intense staining with an intensity score $>2(P=0.0002$, Chi-square, two-sided $)$. In high-grade serous carcinomas, no significant difference in staining intensity or percentage was observed between recurrent and primary tumors based on different cutoffs of intensity score and percentage of pChk2 positive cells. In contrast to carcinoma tissues, ovarian surface epithelium, ovarian surface inclusion cysts, serous cystadenomas, serous borderline tumors, and tumor stromal tissues showed undetectable levels of pChk2 immunoreactivity (Figure 3). There was no significant association of pChk2 expression (intensity or percentage) and clinical outcome, including disease-free survival and overall survival, based on Kaplan-Meier survival analysis in high-grade serous carcinomas (data not shown).

We then tested the correlation between the expression levels of pChk2 and Rsf-1 (HBXAP), because we have recently shown that amplification and overexpression of Rsf1 (HBXAP) contribute to chromosomal instability by inducing DNA strand breaks [20]. Rsf-1 immunostaining results were available in 75 primary high-grade serous carcinomas from our previous study [17], enabling correlation with pChk2 data. Because Rsf-1 immunoreactivity is usually homogenous, we used intensity scores for Rsf- 1 expression as previously described [17]. Rsf-1 immunostaining intensity significantly correlated with both percentage $(P<0.0001)$ and intensity $(P<0.0001)$ of pChk2 (Figures $4(\mathrm{a})$ and $4(\mathrm{~b}))$. Representative immunostained tumor sections of pChk 2 and Rsf- 1 from the same tissues are shown in Figure 4(c).

\section{Discussion}

It has been well established that DNA damage in cancer cells and associated DNA damage response pathway activation 
OSE
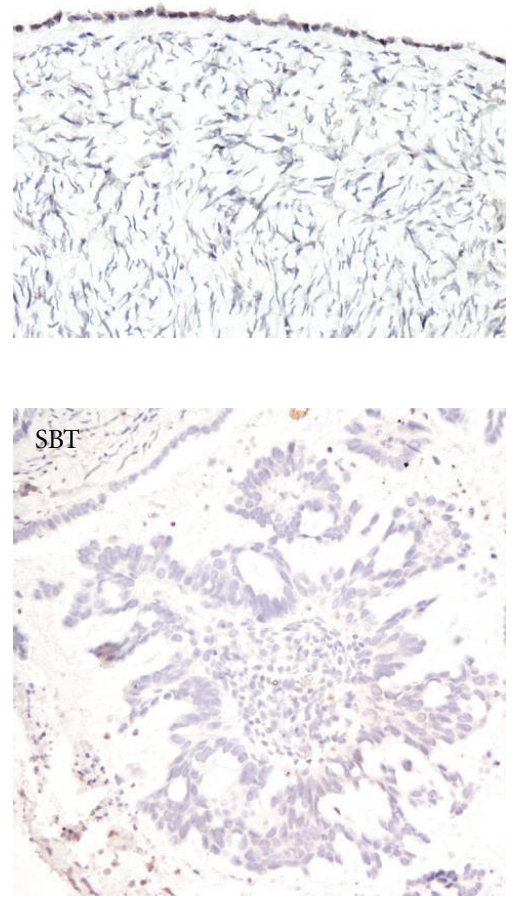

Cyst

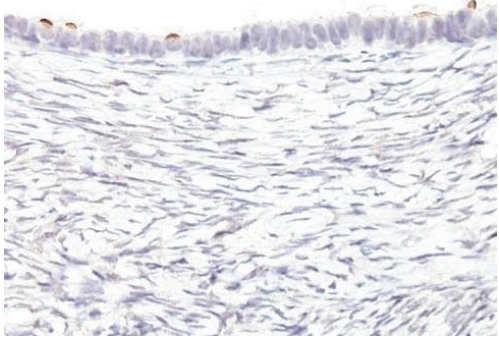

(a)

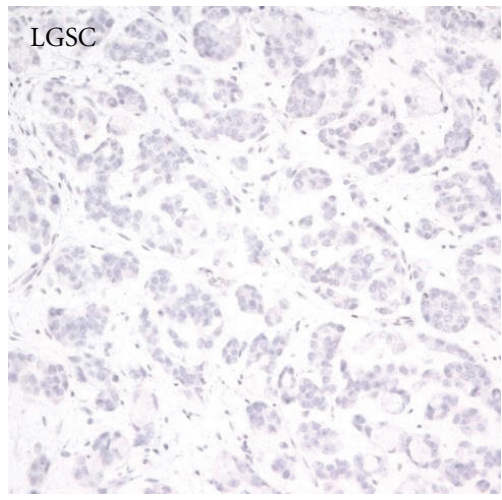

(b)
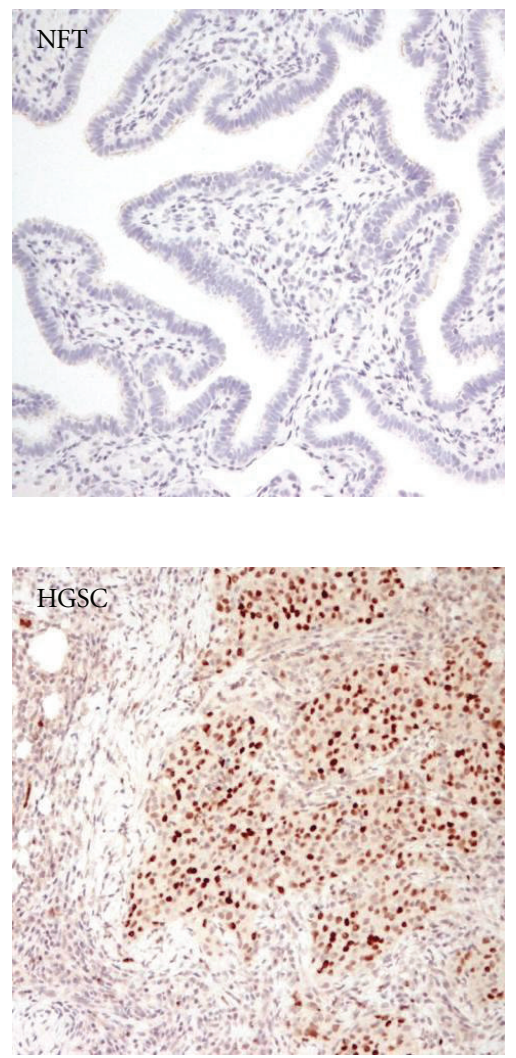

FIGURE 3: pChk2 immunoreactivity in ovarian tissues and different histologic subtypes of ovarian carcinomas. (a) pChk2 staining is undetectable in ovarian surface epithelium (OSE), cystadenoma (Cyst), normal fallopian tube (NFT). (b) serous borderline tumor (SBT) and low-grade serous carcinoma (LGSC), are negative for pChk2 staining. In contrast, a high-grade serous carcinoma (HGSC) is diffusely positive for pChk2.

play an important role in chromosomal instability and tumor development [21-24]. The results from this study underscore the fundamental molecular differences between high-grade and low-grade serous carcinomas in terms of DDR activation. We have previously proposed that highgrade and low-grade serous carcinomas develop along distinct pathways [3]. Low-grade serous carcinoma develops from the precursor lesion, serous borderline tumor, tends to present at early stages, and is slow growing. In contrast, highgrade serous carcinoma, which has been generally referred to as ovarian cancer, behaves in a highly aggressive fashion, almost always present at advanced stages, and is associated with a dismal clinical outcome. They are typically not associated with borderline tumors, and in fact, a growing body of evidence has supported the view that many high-grade serous carcinomas develop from serous tubal intraepithelial carcinomas and involve the ovary secondarily [25-29]. At the molecular genetic level, low-grade serous carcinomas are characterized by frequent somatic sequence mutations in genes that are involved in signal transduction including KRAS, BRAF, ERBB2, and PIK3CA [1, 30-33]. In contrast, mutations in those genes are rarely detected in high-grade serous carcinomas; however, almost all high-grade serous carcinomas harbor TP53 mutations. In addition to unique sequence mutations, high-grade serous carcinomas and lowgrade serous carcinomas (the prototype of type I tumors) have distinct transcriptome and methylation profiles $[3,34$, 35].

The higher pChk2 expression levels in high-grade serous carcinomas suggest that DDR is prominent in high-grade serous carcinomas as compared to low-grade serous carcinomas. This is likely due to frequent DNA damage in highgrade serous carcinomas. The pronounced DDR in highgrade serous carcinomas may be related to DNA replication stress due to activation of oncogenes and telomere shortening among several others [36, 37]. Furthermore, highgrade serous carcinomas usually have higher proliferative activity than low-grade serous carcinomas, precipitating the effects of DNA replication stress. On the other hand, tumor microenvironmental changes such as oxidative stress, hypoxia, and the presence of cytotoxic agents may potentiate DNA damage. Furthermore, recent evidence has suggested that excessive remodeling of chromosomal structures can be related to DNA strand breaks followed by DDR. In fact, we have recently shown that overexpression of a chromatin remodeling gene, Rsf-1 (HBXAP), leads to DNA doublestrand breaks and DDR, resulting in p53-depdenent cell cycle arrest and apoptosis in TP53 wild type, nontransformed 


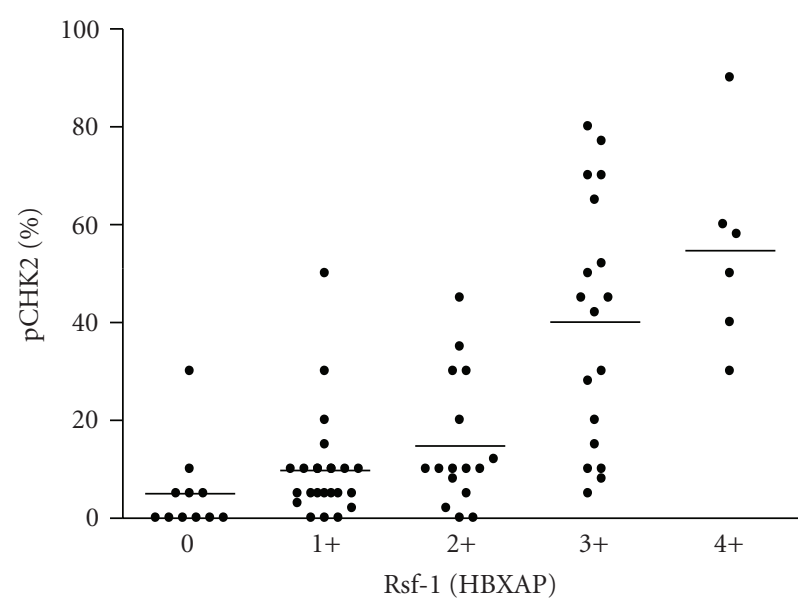

(a)

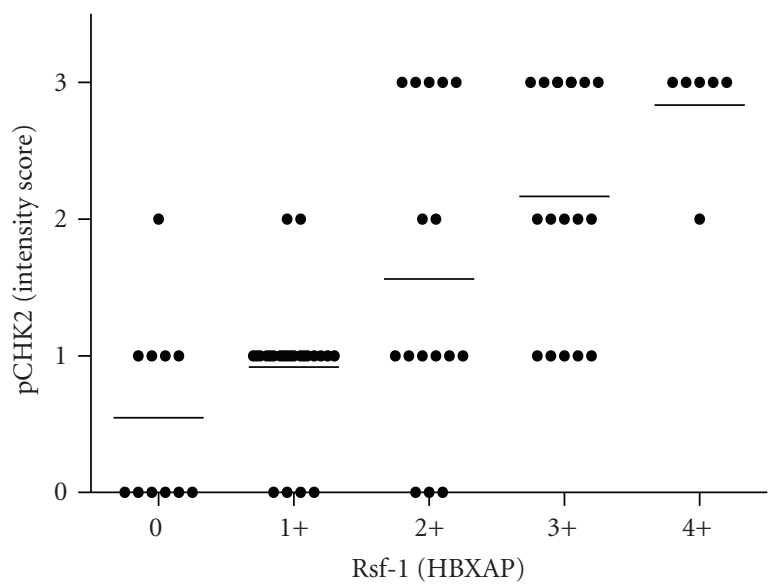

(b)

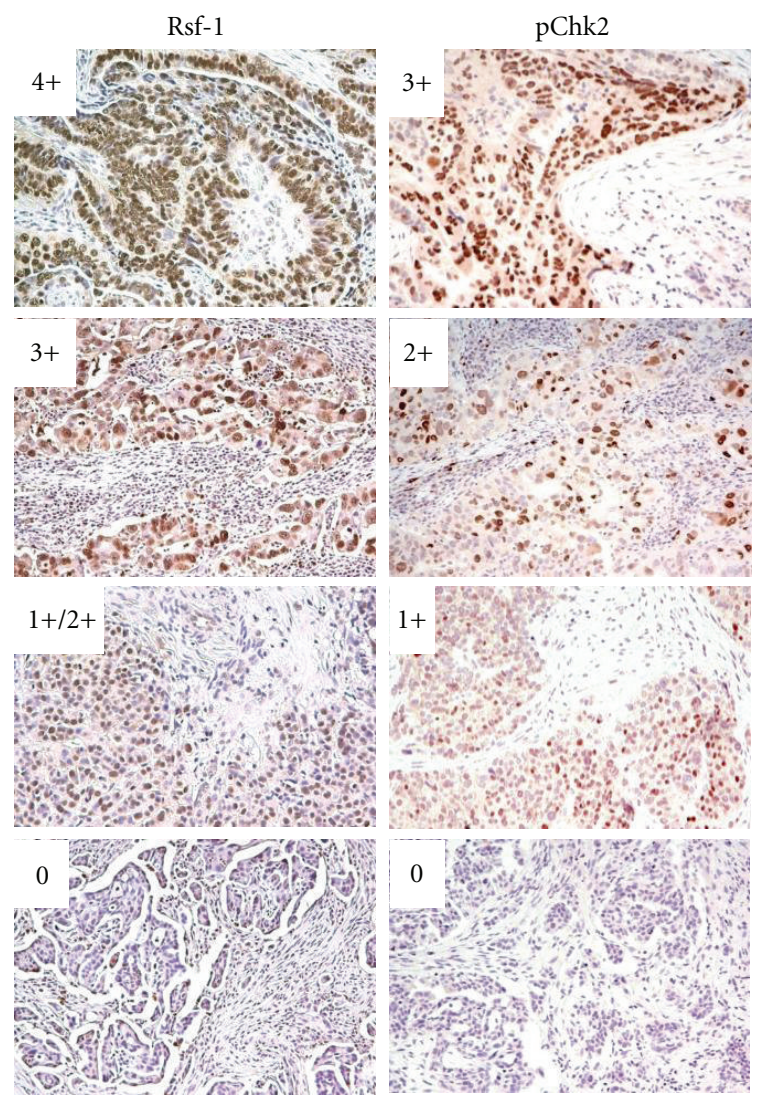

(c)

FIGURE 4: Correlation of the expression levels of pChk2 and Rsf-1 (HBXAP). (a) Scatter plots showing the distribution of pChk2 (percentage) and Rsf-1 (HBXAP) (immunostaining intensity) in 75 high-grade serous carcinomas. The correlation between the percentage of pChk2 positive cells and Rsf-1 immunostaining intensity was significant $(P<0.0001)$. Horizontal bars: means. (b) Scatter plots showing the distribution of pChk2 intensity scores and Rsf-1 (HBXAP) intensity scores in the same set of high-grade serous carcinomas. The correlation between immunostaining intensity of pChk2 and Rsf-1 was significant $(P<0.0001)$. Horizontal bars: means. (c) Four representative cases of high-grade serous carcinomas showing different staining patterns of pChk2 and Rsf- 1 . The intensity scores are shown in the left upper corner of each panel.

cells [20]. DNA damage goes unchecked, and cells continue proliferating in the presence of DNA strand breaks if TP53 is mutated, as what occurs in the great majority of highgrade serous carcinomas. Thus, the result from the current immunohistochemistry study further supports the biological link between Rsf- 1 upregulation and DDR in ovarian cancer tissues either by aberrant chromatin remodeling or by oncogene/replicative stress. As TP53 mutation represents one 
of the very early event in the development of high-grade serous carcinoma, it is currently not clear if DNA damage occurs before or after TP53 mutations, although it has been suggested that activation of Chk2 and other DDR members may precede p53 inactivation in human epithelial tumors $[22,38]$.

It has been demonstrated that high-grade serous carcinoma, as compared to low-grade serous carcinoma, exhibits a higher level of chromosomal instability as reflected by abnormal mitosis, DNA copy number alterations, and karyotypic abnormalities [39, 40]. Thus, the increased pChk2 immunoreactivity in high-grade serous carcinoma raises a possibility that in high-grade serous carcinoma cells, DDR may not be fully capable of repairing DNA damage and maintaining genomic integrity, as the DNA damage may overwhelm the DNA repair capacity. As a result, clonal selection favors tumor cells harboring sustained DNA damage at a level that allows cells to survive but at the same time causes chromosomal instability, serving as the driving force in the evolution of highly aggressive tumor cell species. The finding of pChk2 expression in the majority of high-grade serous carcinomas implies that it may serve as a therapeutic target because inhibiting Chk2 may reduce DNA repair and increase genomic instability to a level that is not compatible with cellular survival and ultimately leads to tumor cell death. Indeed, it has been recently reported that a newly developed Chk2 small compound inhibitor could potentiate the cytotoxicity effect of PARP inhibitors, a finding supporting this view [41].

The majority of low-grade serous carcinomas do not contain prominent pChk2 immunoreactivity. Although our favored view is that less DNA damage is present, and, thus, attenuated DDR occurs in those tumors, other interpretations should also be pointed out. For example, the lack of pChk2 immunoreactivity might be a consequence of inactivation of upstream components in the DDR pathway [36]. As a result, ineffective DDR is present despite DNA damage in tumor cells. However, this scenario is less likely because unchecked and unrepaired DNA damage is generally detrimental to cells and is incompatible with sustained survival and proliferation in tumor cells.

In summary, using pChk2 immunoreactivity as a surrogate marker for DDR, we found that high level DDR was detected more frequently in high-grade serous carcinomas than in low-grade serous carcinoma. This finding provides further support to the view that both tumors are molecularly distinct and develop along different molecular pathways. The significant correlation of expression between pChk2 and Rsf1 (HBXAP) suggests that excessive chromatin remodeling activity as a result of upregulation of Rsf-1 (HBXAP) is associated with DDR in high-grade serous carcinoma tissues, a result supporting our previous observations that Rsf-1 upregulation contributes to DNA strand breaks and subsequent DDR. Future studies should aim at deciphering the mechanisms responsible for prominent DNA damage in highgrade serous carcinomas as compared to low-grade serous carcinomas and perhaps other type I tumors. Although our current study did not demonstrate an association of pChk2 expression levels with overall survival and disease-free sur- vival, future clinical studies should be conducted to assess the biological significance of pChk2 in ovarian cancer patients.

\author{
Abbreviations \\ Ch: Chromosome \\ Chk2: Check point kinase 2 \\ DDR: DNA damage response.
}

\section{Authors' Contribution}

Malti Kshirsagar and Wei Jiang contribute equally to this work.

\section{Acknowledgment}

This study was supported by an NIH/NCI Grant, CA129080.

\section{References}

[1] K. R. Cho and I. M. Shih, "Ovarian cancer," Annual Review of Pathology, vol. 4, pp. 287-313, 2009.

[2] M. Köbel, S. E. Kalloger, N. Boyd et al., "Ovarian carcinoma subtypes are different diseases: implications for biomarker studies," PLoS Medicine, vol. 5, no. 12, article e232, 2008.

[3] I. M. Shih and R. J. Kurman, "Ovarian tumorigenesis: a proposed model based on morphological and molecular genetic analysis," American Journal of Pathology, vol. 164, no. 5, pp. 1511-1518, 2004.

[4] K. Oka, T. Tanaka, T. Enoki et al., "DNA damage signaling is activated during cancer progression in human colorectal carcinoma," Cancer Biology and Therapy, vol. 9, no. 3, pp. 246-252, 2010.

[5] J. B. M. Koorstra, S. M. Hong, C. Shi et al., "Widespread activation of the DNA damage response in human pancreatic intraepithelial neoplasia," Modern Pathology, vol. 22, no. 11, pp. 1439-1445, 2009.

[6] A. J. Yoon, J. Shen, R. M. Santella, D. J. Zegarelli, R. Chen, and I. B. Weinstein, "Activated checkpoint kinase 2 expression and risk for oral squamous cell carcinoma," Cancer Epidemiology Biomarkers and Prevention, vol. 16, no. 12, pp. 2768-2772, 2007.

[7] F. Al-Ejeh, R. Kumar, A. Wiegmans, S. R. Lakhani, M. P. Brown, and K. K. Khanna, "Harnessing the complexity of DNA-damage response pathways to improve cancer treatment outcomes," Oncogene, vol. 29, no. 46, pp. 6085-6098, 2010.

[8] J. W. Harper and S. J. Elledge, "The DNA damage response: ten years after," Molecular Cell, vol. 28, no. 5, pp. 739-745, 2007.

[9] T. H. Stracker, T. Usui, and J. H. J. Petrini, "Taking the time to make important decisions: the checkpoint effector kinases Chk1 and Chk2 and the DNA damage response," DNA Repair, vol. 8, no. 9, pp. 1047-1054, 2009.

[10] S. Matsuoka, M. Huang, and S. J. Elledge, "Linkage of ATM to cell cycle regulation by the Chk2 protein kinase," Science, vol. 282, no. 5395, pp. 1893-1897, 1998.

[11] J. Falck, N. Mailand, R. G. Syljuåsen, J. Bartek, and J. Lukas, "The ATM-Chk2-Cdc25A checkpoint pathway guards against radioresistant DNA synthesis," Nature, vol. 410, no. 6830, pp. 842-847, 2001.

[12] I. M. Ward, X. Wu, and J. Chen, "Threonine 68 of Chk2 is phosphorylated at sites of DNA strand breaks," Journal of Biological Chemistry, vol. 276, no. 51, pp. 47755-47758, 2001. 
[13] A. J. Yoon, J. Shen, H. C. Wu et al., "Expression of activated checkpoint kinase 2 and histone 2AX in exfoliative oral cells after exposure to ionizing radiation," Radiation Research, vol. 171, no. 6, pp. 771-775, 2009.

[14] K. Stefanidis, D. Loutradis, L. V. Vassiliou et al., "Nevirapine induces growth arrest and premature senescence in human cervical carcinoma cells," Gynecologic Oncology, vol. 111, no. 2, pp. 344-349, 2008.

[15] R. A. DiTullio, T. A. Mochan, M. Venere et al., "53BP1 functions in a ATM-dependent checkpoint pathway that is constitutively activated in human cancer," Nature Cell Biology, vol. 4, no. 12, pp. 998-1002, 2002.

[16] I. M. Shih, J. J. C. Sheu, A. Santillan et al., "Amplification of a chromatin remodeling gene, Rsf-1/HBXAP, in ovarian carcinoma," Proceedings of the National Academy of Sciences of the United States of America, vol. 102, no. 39, pp. 14004-14009, 2005.

[17] T. L. Mao, C. Y. Hsu, M. J. Yen et al., "Expression of Rsf-1, a chromatin-remodeling gene, in ovarian and breast carcinoma," Human Pathology, vol. 37, no. 9, pp. 1169-1175, 2006.

[18] K. Nakayama, N. Nakayama, N. Jinawath et al., "Amplicon profiles in ovarian serous carcinomas," International Journal of Cancer, vol. 120, no. 12, pp. 2613-2617, 2007.

[19] C. Lukas, J. Falck, J. Bartkova, J. Bartek, and J. Lukas, “Distinct spatiotemporal dynamics of mammalian checkpoint regulators induced by DNA damage," Nature Cell Biology, vol. 5, no. 3, pp. 255-260, 2003.

[20] J. J. C. Sheu, B. Guan, J. H. Choi et al., "Rsf-1, a chromatin remodeling protein, induces DNA damage and promotes genomic instability," Journal of Biological Chemistry, vol. 285, no. 49 , pp. 38260-38269, 2010.

[21] V. G. Gorgoulis, L. V. F. Vassiliou, P. Karakaidos et al., "Activation of the DNA damage checkpoint and genomic instability in human precancerous lesions," Nature, vol. 434, no. 7035, pp. 907-913, 2005.

[22] J. Bartkova, Z. Hořejší, K. Koed et al., "DNA damage response as a candidate anti-cancer barrier in early human tumorigenesis," Nature, vol. 434, no. 7035, pp. 864-870, 2005.

[23] J. Bartkova, N. Rezaei, M. Liontos et al., "Oncogene-induced senescence is part of the tumorigenesis barrier imposed by DNA damage checkpoints," Nature, vol. 444, no. 7119, pp. 633-637, 2006.

[24] R. Di Micco, M. Fumagalli, A. Cicalese et al., "Oncogeneinduced senescence is a DNA damage response triggered by DNA hyper-replication," Nature, vol. 444, no. 7119, pp. 638642, 2006.

[25] D. W. Kindelberger, Y. Lee, A. Miron et al., "Intraepithelial carcinoma of the fimbria and pelvic serous carcinoma: evidence for a causal relationship," American Journal of Surgical Pathology, vol. 31, no. 2, pp. 161-169, 2007.

[26] C. P. Crum, R. Drapkin, A. Miron et al., "The distal fallopian tube: a new model for pelvic serous carcinogenesis," Current Opinion in Obstetrics and Gynecology, vol. 19, no. 1, pp. 3-9, 2007.

[27] R. J. Kurman and I. M. Shih, "The origin and pathogenesis of epithelial ovarian cancer: a proposed unifying theory," American Journal of Surgical Pathology, vol. 34, no. 3, pp. 433-443, 2010.

[28] K. Visvanathan, A. L. Gross, R. J. Kurman, R. Vang, and I. M. Shih, "Precursor lesions of high-grade serous ovarian carcinoma: morphological and molecular characteristics," Journal of Oncology, Article ID 126295, 2010.

[29] S. Salvador, B. Gilks, M. Köbel, D. Huntsman, B. Rosen, and D. Miller, "The fallopian tube: primary site of most pelvic high-grade serous carcinomas," International Journal of Gynecological Cancer, vol. 19, no. 1, pp. 58-64, 2009.

[30] G. Singer, R. J. Kurman, H. W. Chang, S. K. R. Cho, and I. M. Shih, "Diverse tumorigenic pathways in ovarian serous carcinoma," American Journal of Pathology, vol. 160, no. 4, pp. 1223-1228, 2002.

[31] G. Singer, R. Oldt III, Y. Cohen et al., "Mutations in BRAF and KRAS characterize the development of low-grade ovarian serous carcinoma," Journal of the National Cancer Institute, vol. 95, no. 6, pp. 484-486, 2003.

[32] K. Nakayama, N. Nakayama, R. J. Kurman et al., "Sequence mutations and amplification of PIK3CA and AKT2 genes in purified ovarian serous neoplasms," Cancer Biology and Therapy, vol. 5, no. 7, pp. 779-785, 2006.

[33] S. Jones, T. L. Wang, I. M. Shih et al., "Frequent mutations of chromatin remodeling gene ARID1A in ovarian clear cell carcinoma," Science, vol. 330, no. 6001, pp. 228-231, 2010.

[34] I. Meinhold-Heerlein, D. Bauerschlag, F. Hilpert et al., "Molecular and prognostic distinction between serous ovarian carcinomas of varying grade and malignant potential," Oncogene, vol. 24, no. 6, pp. 1053-1065, 2005.

[35] I.-M. Shih, L. Chen, C. C. Wang et al., "Distinct DNA methylation profiles in ovarian sreous neoplasms and their implications in ovarian carcinogenesis," American Journal of Obstetrics \& Gynecology, vol. 203, no. 6, pp. 584.e1-584.e22, 2010.

[36] C. Kerzendorfer and M. O’Driscoll, "Human DNA damage response and repair deficiency syndromes: linking genomic instability and cell cycle checkpoint proficiency," DNA Repair, vol. 8, no. 9, pp. 1139-1152, 2009.

[37] D. Lydall, "Taming the tiger by the tail: modulation of DNA damage responses by telomeres," EMBO Journal, vol. 28, no. 15, pp. 2174-2187, 2009.

[38] T. D. Halazonetis, V. G. Gorgoulis, and J. Bartek, "An oncogene-induced DNA damage model for cancer development," Science, vol. 319, no. 5868, pp. 1352-1355, 2008.

[39] K. T. Kuo, T. L. Mao, Y. Feng et al., "DNA copy numbers profiles in affinity-purified ovarian clear cell carcinoma," Clinical Cancer Research, vol. 16, no. 7, pp. 1997-2008, 2010.

[40] K. T. Kuo, B. Guan, Y. Feng et al., "Analysis of DNA copy number alterations in ovarian serous tumors identifies new molecular genetic changes in low-grade and high-grade carcinomas," Cancer Research, vol. 69, no. 9, pp. 4036-4042, 2009.

[41] V. E. Anderson, M. I. Walton, P. D. Eve et al., "CCT241533 is a potent and selective inhibitor of $\mathrm{CHK} 2$ that potentiates the cytotoxicity of PARP inhibitors," Cancer Research, vol. 71, no. 2, pp. 463-472, 2011. 


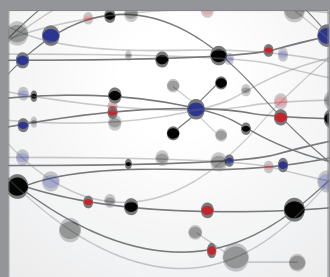

The Scientific World Journal
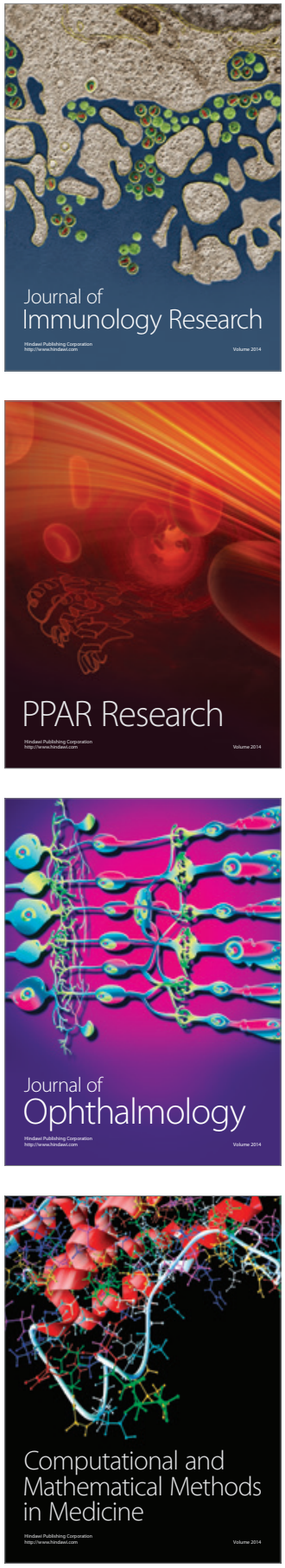

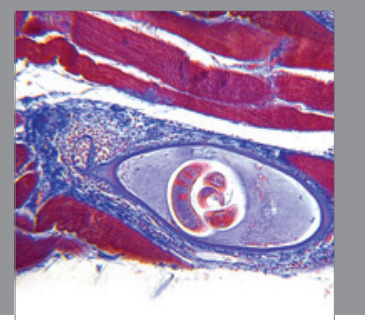

Gastroenterology

Research and Practice
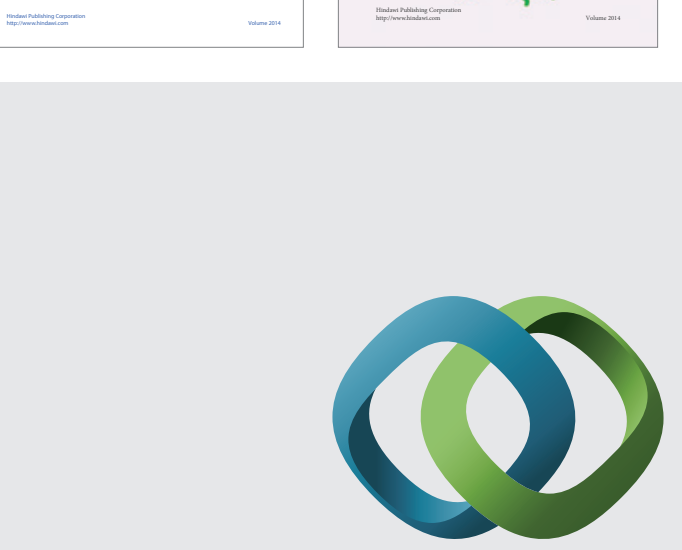

\section{Hindawi}

Submit your manuscripts at

http://www.hindawi.com
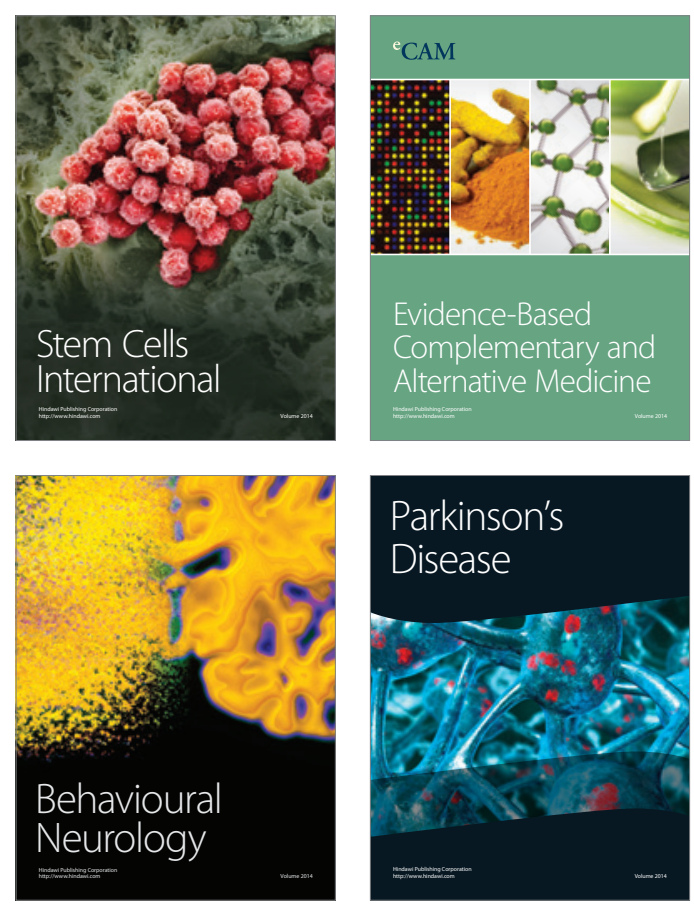

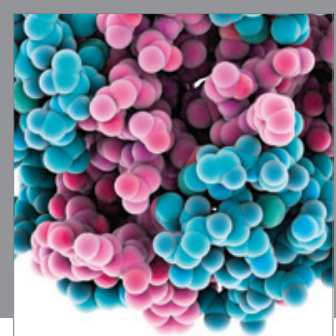

Journal of
Diabetes Research

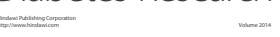

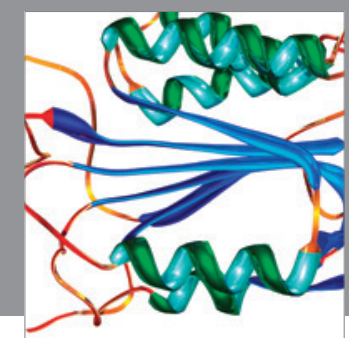

Disease Markers
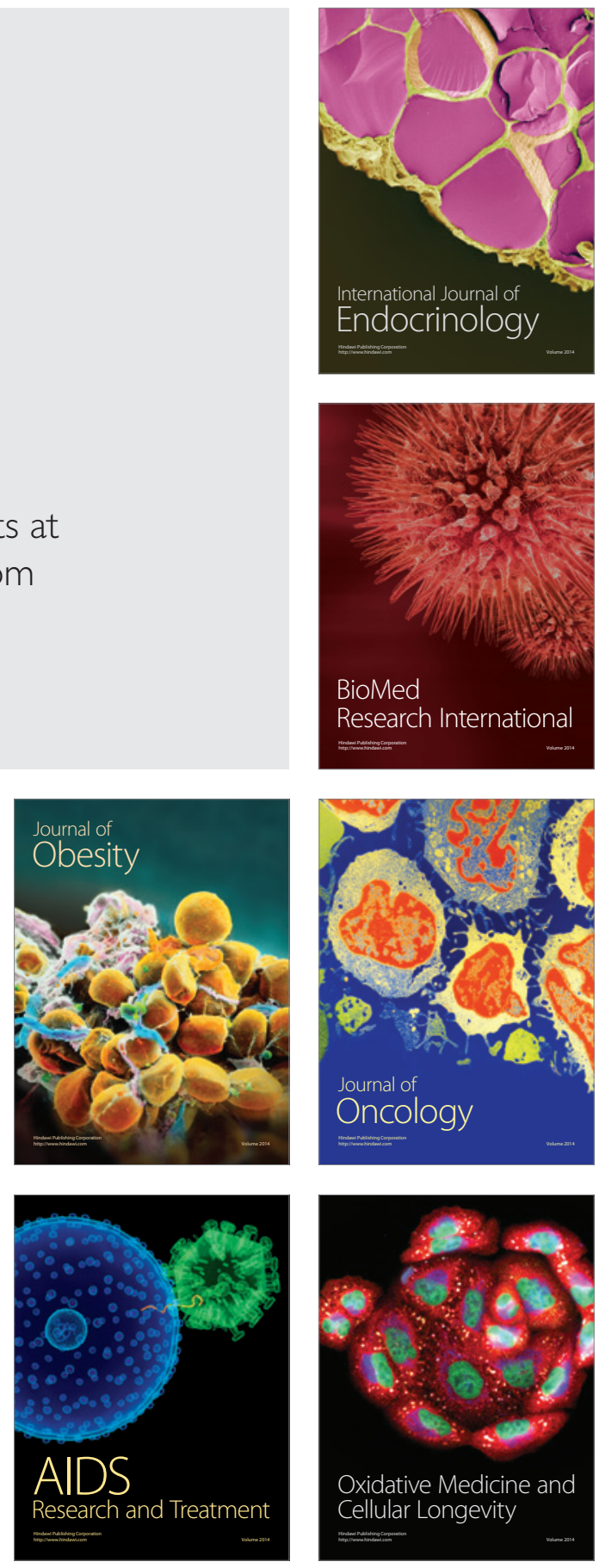